\title{
Unusual conductance of polyyne-based molecular wires
}

\author{
Ž. Crljer* and G. Baranović \\ R. Bošković Institute, P.O. Box 180, 10002 Zagreb, Croatia
}

(Dated: May 4, 2006)

\begin{abstract}
We report a full self-consistent $a b$ initio calculation of the current-voltage curve and the conductance of thiolate capped polyynes in contact with gold electrodes. We find the conductance of polyynes an order of magnitude larger compared with other conjugated oligomers. The reason lies in the position of the Fermi level deep in the HOMO related resonance. With the conductance weakly dependent on the applied bias and almost independent of the length of the molecular chain, polyynes appear as nearly perfect molecular wires.

PACS numbers: 85.65.+h; 73.63.-b; 71.15.Mb

Keywords:
\end{abstract}

The study of transport properties of single molecules have attracted a significant attention because of their potential use in molecular electronic devices. One of the major classes of molecules considered in conductivity studies, primarily for their molecular wire behavior, 1.2 .3 .4 .5 is conjugated oligomers. They have shown a number of useful nonlinear properties such as conductance switching and negative differential resistance ${ }^{6.7}$ However, inspite of a number of interesting experiments 4.8 a molecule with good molecular wire properties has not yet been spotted.

A useful molecular wire should provide a high and stable conductance over a wide bias region and for various lengths of the molecules. A linear chain of carbon atoms with double bonds between neighboring atoms, usually referred to as cumulene, was proposed as an ideal molecular wire $\underline{\underline{\underline{1}}}$ and the calculations of the conductance of cumulene connected to gold electrodes were reported ${ }^{9.10}$ Lang and Avouris ${ }^{9}$ showed that the conductance of cumulene did not stay constant in the ballistic regime, but rather oscillated between the constant values characteristics of the odd and even number of atoms in the chain.

In this paper we show an entirely different behavior of polyynes, another form of the carbon atom chain. Polyynes are simple and yet most intriguing of conjugated organic oligomers. Only recently, have they been assembled up to decayne 11 Formed as a linear chain of carbon atom pairs $(C C)_{n}$, with alternating single and triple bonds, they are a unique, truly one-dimensional, molecular system. Two $\pi$-electron systems of the sphybridized structure provide polyyne with approximately cylindrical electronic delocalization along the conjugated backbone. The electronic transport is therefore independent of the rotation around the single bond, which is a limitation often present in other organic oligomers ${ }^{?}$

We have obtained the electronic structure and transport properties of a series of polyynes up to octayne, connected to gold electrodes. The stability of polyyne with respect to single- and triple-bond alternations was achieved by fixing the molecule at the ends with thiol bonds. In addition, the thiol capped polyynes make a strong chemisorption bond onto the metallic electrodes. We found that they had more than an order of mag- nitude higher conductance when compared with other conjugated oligomers. In contrast to the cumulenes they were not prone to oscillations in conductance with the length of the molecule. We also found that their conductance was very weakly dependent on the molecular length and almost constant in the wide range of bias voltages.

A previous study, with a different theoretical approach, included polyynes up to triyne with Pd contacts of a different contact geometry which resulted in lower conductance 12

In order to perform the first-principle quantum modeling of the electronic structure under nonequilibrium conditions and to calculate the current-voltage characteristics and the differential conductance of the system, a full self-consistent $a b$ initio method, which includes portions of the electrodes, had to be used ${ }^{13}$ The calculations were carried out using a nonequilibrium Green functions technique based on density functional theory, as implemented in the TranSIESTA simulation package 14 The current through the contact was calculated using the LandauerButtiker formula, $\frac{15}{5} I\left(V_{b}\right)=G_{0} \int_{\mu_{L}}^{\mu_{R}} T\left(E, V_{b}\right) d E$, where $G_{0}=2 e^{2} / h$ is the quantum unit of conductance and $T\left(E, V_{b}\right)$ is the transmission probability for electrons incident at an energy $E$ through the device under the potential bias $V_{b}$. The difference between the electrochemical potentials $\mu_{L / R}$, of the left and right electrodes, respectively, is $\mu_{L}-\mu_{R}=e V_{b}$. The computational procedure used was described extensively elsewhere ${ }^{5}$.

The molecular electronic system considered consisted of a monolayer of molecules coupled to two semi-infinite electrodes, as depicted in Fig 1 for hexayne. We optimized the geometry of free thiol capped polyyne in a separate DFT calculation ${ }^{16}$. The molecule was then positioned perpendicularly to the z-direction in the hollow sites of both $\mathrm{Au}(111)$ surfaces of the electrodes symmetrically at a favorable $\mathrm{Au}-\mathrm{S}$ bonding distance $\frac{17}{}$. We let the molecular coordinate relax, keeping the gold atoms at their bulk positions. A small change in the molecular geometry occurred with respect to the free molecule. We discuss this at the end of the paper.

The main characteristics of the obtained transmission spectra are the almost linear increase of the current with bias voltage and a high value of conductance over a wide 


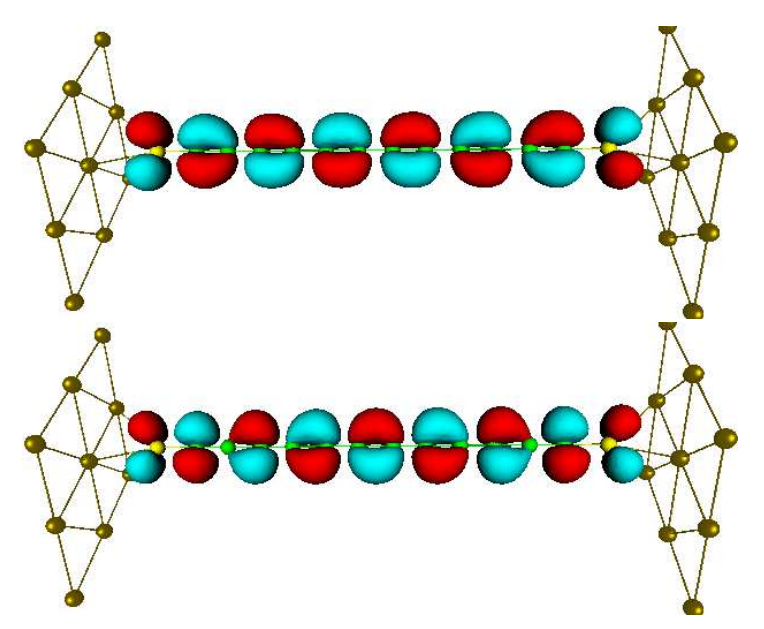

FIG. 1: Hexayne connected to two $\mathrm{Au}(111)$ surfaces via thiolate bonds, shown with the MPSH HOMO orbital (upper panel, notice the delocalized shape at each side of single bond) and the MPSH LUMO orbital (lower panel) at $V_{b}=0.6 \mathrm{~V}$ bias voltage.

bias region from $-2 \mathrm{~V}$ to $2 \mathrm{~V}$, as seen in Fig 2 The obtained spectra of all members 18 of the polyyne series show a mutual pronounced similarity without oscillations with the length of the molecule. The conductance at zero bias voltage is $1.65 G_{0}$ for diyne, $1.56 G_{0}$ for tetrayne, 1.49 $G_{0}$ for hexayne, and $1.44 G_{0}$ for octayne. The overall high value of conductance is expected, since the main transmission channels involve double degenerate molecular $\pi$ orbitals. Unlike in the true ballistic transport in a quantum structure where a constant conductance might be expected irrespective of the molecular length ${ }^{19}$, a slow decrease of conductance with the length of the molecule is demonstrated. The decrease should be attributed to a weak reduction of hybridization at the molecule-electrode contact with the number of atoms in the molecular chain 20 .

When compared with other conjugated oligomers, polyynes show much higher conductance. In Fig 3 we compare the transmission amplitude at zero bias of three different thiolate capped molecules: hexayne, diphenyl diacetylene (DPA2), and phenylene vinylene oligomer with three benzene rings (OPV3). They were all chemisorbed onto $\mathrm{Au}(111)$ electrodes in hollow positions at both ends in order to ensure the same bonding geometry thus avoiding the possible bonding site effects on the considered transmission. The resulting interelectrode separations for all three systems were rather similar, but the corresponding zero bias conductances differed by more than an order of magnitude, as shown in Table [1. The large difference in conductance is a consequence of the entirely different electronic structure and density of states at the Fermi level $\left(E_{f}\right)$. The hybridization of the molecular level with the gold electrode states broadens the level into the resonance. The width and the position of the resonance with respect to the Fermi level of the system depend on the internal structure of the molecule

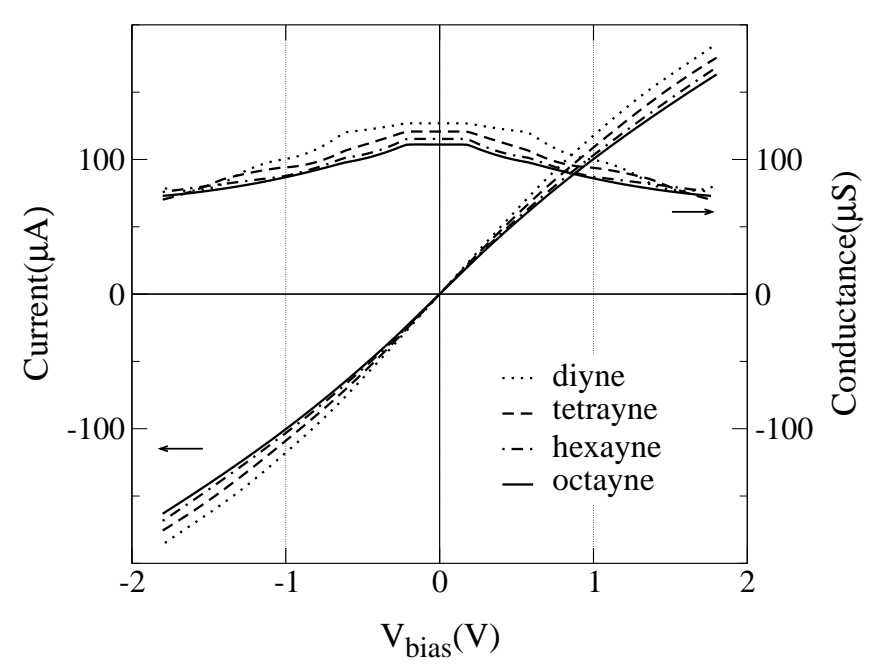

FIG. 2: Current and differential conductance of polyyne systems as a function of bias voltage. A self-consistent calculation has been employed for each bias voltage. Notice the slow and smooth almost linear decrease of the conductance.

and its bonding to the electrode. In the hexayne case, the double degenerate molecular $\pi$ orbitals are involved. The resulting HOMO related resonance ${ }^{21}$ is wide and shifted up in energy close to the Fermi level, as seen in Fig 3, In fact, the HOMO and LUMO related resonances strongly overlap giving a wide transmission band with large density of states, which results in high transmission. The transmission of DPA2 and OPV3 systems is considerably smaller when compared with the hexayne, as shown in Fig 3 The reason for that is the lifted HOMO level degeneracy in DPA2 and OPV3 and the positioning of the HOMO level further below $E_{f}$, which results in the lower density of states at the Fermi level.

The high density of states at the Fermi level is the property of other members of the polyyne family, as well. With the increased length of the polyyne chain, levels are becoming more densely distributed and of shorter width. The remarkable property is, however, that the position of the HOMO related resonance moves slightly closer to $E_{f}$, thus compensating for the decrease of the density of states at $E_{f}$ due to the level sharpness, as seen in Fig 4 That results in a very weak dependence of the zero bias conductance on the molecular length.

The conductance of polyynes decreases smoothly with the increase of the bias voltage, as shown in Fig 2 In or-

TABLE I: Interelectrode separation $d_{\text {elec }}$ and zero-bias conductance $\mathrm{G}(\mu, 0)$ of polyyne systems compared with molecules of similar length. $d_{\text {elec }}$ is the distance between the surface gold planes of the electrodes.

\begin{tabular}{cccc}
\hline \hline & OPV3 & DPA2 & hexayne \\
\hline$d_{\text {elec }}(n m)$ & 2.3 & 1.95 & 2.127 \\
$\mathrm{G}(\mu, 0)(\mu S)$ & 2.1 & 6.12 & 111.1 \\
\hline \hline
\end{tabular}




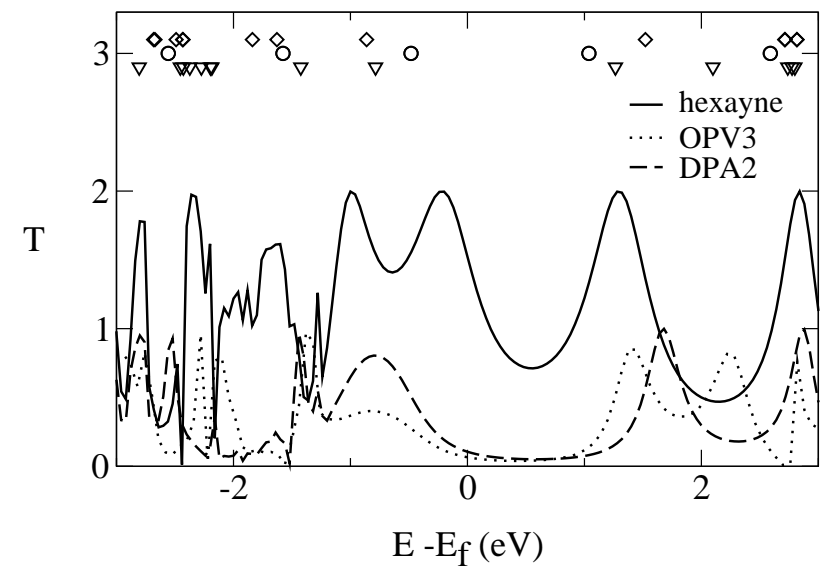

FIG. 3: Transmission amplitude of hexayne compared with DPA2 and OPV3 at zero bias voltage. MPSH eigenvalues are marked with circles for hexayne, diamonds for DPA2, and triangles for OPV3.

der to elucidate this behavior, we show the transmission amplitudes of hexayne for a set of bias values in Fig 5 A remarkable similarity, in both shape and value, of transmission followed by a slight shift of the position of LUMO and higher resonances with the bias is seen. The separation between HOMO and LUMO levels decreases only by $0.11 \mathrm{eV}$ at the bias of $1.8 \mathrm{~V}$ with respect to the zero bias value. The consequence of the similarity of transmission spectra is the relatively weak dependence of the conductance of hexayne on the bias, as seen in Fig 2

The other polyynes showed the dependence of transmission amplitudes when subjected to different bias voltages similar to those for hexayne. With the change of the bias from $0 \mathrm{~V}$ to $2.0 \mathrm{~V}$, the HOMO-LUMO energy separation for all polyynes that we consider decreases by no more than $0.15 \mathrm{eV}$. Such a small change in the molecular level positions relative the average electrochemical potential of the electrodes ensures a slow decrease of the conductance over the entire bias region, as clearly seen in Fig 2

What makes polyynes so much different from other conjugated polymeres is the high density of states at the Fermi level. Even in a free molecule of polyyne, where the alternate single and triple bonds open up a HOMO-LUMO gap, the strong polarizability and hyperpolarizability ${ }^{11.22}$ evolve, owing to the large number of electrons. When thiol capped polyynes are chemisorbed onto gold electrodes, the strong hybridization of molecular states with the metallic electrode states results in a new electronic structure of the combined system. The change of the level positions is so strong that the Fermi level of the system enters deep into the HOMO related resonance resulting in the pronounced metallic character of the system.

As already said, we used LDA for the exchangecorrelation functional in our DFT calculations. There has been a discussion in the literature on the validity of

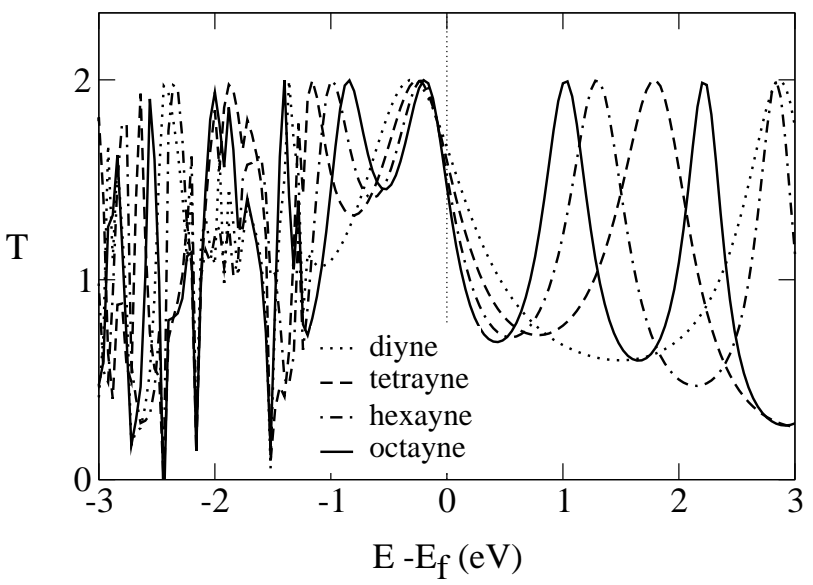

FIG. 4: Transmission amplitude of polyynes at zero bias voltage. Note the rather stable position of HOMO related peaks for all polyynes.

DFT with the local density (LDA) and generalized gradient (GGA) approximations for $\pi$-conjugated systems 23 . Here, however, the $\pi$-conjugated system is connected to the electrodes and shows more metalliclike character. LDA- and GGA-based DFT calculations are therefore expected to give a respectable accuracy usually obtained in DFT calculations. The crucial parameters determining the HOMO-LUMO gap are the carbon-carbon bond lengths and their alternations. We performed the calculations for isolated molecules of thiolate capped polyynes using the Gaussian03 program ${ }^{24}$ with several XC functionals and found that the GGA results did not substantially improve the values of the gap, nor the single and triple bond lengths. Indeed, the difference in the HOMOLUMO gaps was less than $0.037 \mathrm{eV}$ between the PW91 (GGA) and SVWN (LDA) based calculations, as seen in Table III When extrapolated to infinite chain length the gap stayed open, in agreement with the calculations with

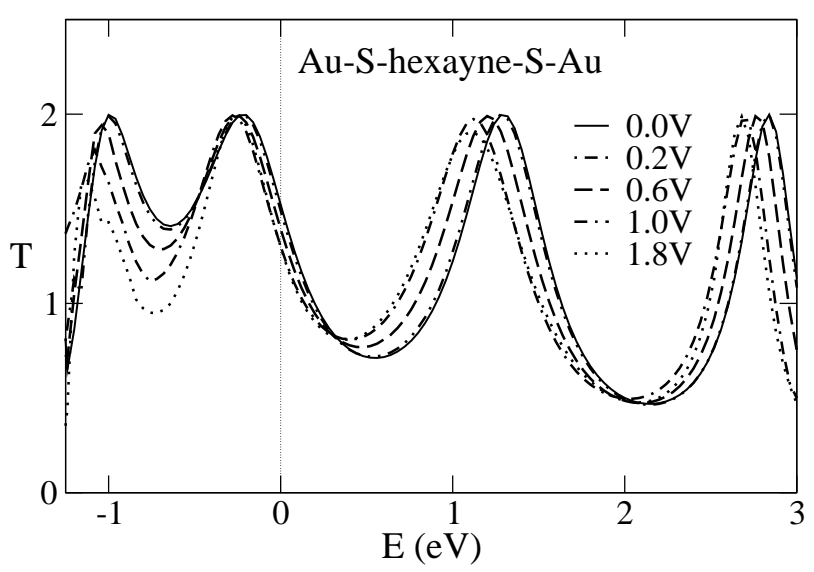

FIG. 5: Transmission amplitude of dithiolhexayne connected to gold electrodes as a function of bias voltage. Energies measured from the average electrochemical potential of the electrodes. 
TABLE II: MPSH HOMO-LUMO energy gap $\Delta E$, in $e V$, at zero bias of thiolate capped polyynes with $\mathrm{Au}$ electrodes compared with the HOMO-LUMO gaps of bare thiol capped polyynes (DFT calculations with SVWN, PW91, and PBE $\mathrm{XC}$ functionals).

\begin{tabular}{cccccccc}
\hline \hline & \multicolumn{3}{c}{$A u S(C C)_{n} S A u$} & & \multicolumn{3}{c}{$H S(C C)_{n} S H$} \\
\cline { 2 - 5 } \cline { 6 - 8 } $\mathrm{n}$ & $\Delta E$ & $E_{H O M O}$ & $E_{L U M O}$ & & $\Delta E_{S V W N}$ & $\Delta E_{P W 91}$ & $\Delta E_{P B E}$ \\
\hline 2 & 3.254 & -0.897 & 2.357 & & 3.529 & 3.559 & 3.549 \\
4 & 2.063 & -0.643 & 1.420 & & 2.333 & 2.370 & 2.364 \\
6 & 1.520 & -0.482 & 1.038 & & 1.762 & 1.797 & 1.788 \\
8 & 1.211 & -0.385 & 0.825 & & 1.428 & 1.463 & 1.458 \\
\hline \hline
\end{tabular}

more exact exchange potentials of Weimer et al ${ }^{25}$ For the molecule bonded to the electrodes, the HOMO-LUMO MPSH gap (first column of Table III) was reduced with respect to the isolated molecule as expected. The reduction was slightly more pronounced for shorter molecules, equal to $0.031 \mathrm{eV}$ for diyne, and decreased to $0.025 \mathrm{eV}$ for octayne. Evidently, the HOMO-LUMO MPSH gap closely followed the gap of the isolated molecule. The possible underestimation of the gap in the LDA-based calculations compared with the nonlocal XC potential calculations would have no major effect. The metalliclike character of the conductance appears to be the consequence of the hybridization of the $\pi$-electron systems of polyynes with the gold electrode states, irrespective of the details of the $\mathrm{XC}$ potential.

The hybridization with the gold states did not affect the bond alternation in the electrode-connected hexayne, as seen in Table TIII The slight reduction of single bonds and the expansion of triple bonds of the connected hexayne are noticed. The change of the bond length is the interplay of the hybridization of the molecular states with the gold electrode states and the relaxation of position of molecular atoms. The amount of change is smaller when the molecule is longer owing to the redistribution of the relaxation over the entire molecule.

TABLE III: Average single and triple related bond distances, measured in $\AA$, of thiolate capped hexayne in contact with $\mathrm{Au}$ electrodes compared with the bare thiol capped hexayne bond distances.

\begin{tabular}{lcccc}
\hline \hline & $A u S(C C)_{6} S A u$ & \multicolumn{3}{c}{$H S(C C)_{6} S H$} \\
\cline { 3 - 5 } & & $S V W N$ & $P W 91$ & $P B E$ \\
\hline$d_{\text {single }}(\AA)$ & 1.316 & 1.321 & 1.330 & 1.332 \\
$d_{\text {triple }}(\AA)$ & 1.286 & 1.249 & 1.252 & 1.253 \\
\hline \hline
\end{tabular}

In this paper, we have shown that polyynes are molecules with high conductance over the large bias region almost irrespective of the length of the molecule considered. Although polyynes form $\pi$-conjugated systems, they show an almost metalliclike character of the current transport, when connected to the electrodes. This makes them a possible candidate for a good molecular wire to be considered in molecular nanoelectronics.

This work was supported in part by the Ministry of Science and Technology of the Republic of Croatia.
* crljen@irb.hr

1 J.S. Schumm, D.L. Pearson, and J.M. Tour, Angew. Chem., Int. Ed. Engl. 33, 1360 (1994).

2 W.B. Davis et al., Nature 396, 60 (1998).

3 M. Magoga and C. Joachim, Phys. Rev. B 56, 4722 (1997);

${ }^{4}$ Getty et al., Phs. Rev B 71, 241401(R) (2005).

5 Z. Crljen, et al., Phys. Rev. B 71, 165316 (2005).

6 J. Chen et al., Science 286, 1550 (1999); M.A. Reed et al., Appl. Phys. Lett. 78, 3735 (2001).

7 J. Chen and M.A. Reed, Chem. Phys. 281, 127 (2002); J. Taylor, M. Brandbyge and K. Stokbro, Phys. Rev. B 68, 121101(R) (2003).

${ }^{8}$ C. Kergueris et al., Phys. Rev. B 59, 12505 (1999); C. Joachim, J.K. Gimzewski and A. Aviram, Nature 408, 541 (2000); J. Reichert, J.K. Gimzewski and A. Aviram, Nature 408, 541 (2000); X.D. Cui et al., Science 294, 571 (2001).

9 N. D. Lang and Ph. Avouris, Phys. Rev. Lett. 81, 3515 (1998); ibid. 84, 358 (2000).

10 M. Brandbyge et al., Phys. Rev. B 65, 165401, (2002).

11 A.D. Slepkov et al., J. Chem. Phys. 120, 6807 (2004) and references therein.

12 J.M. Seminario et al., J. Phys. Chem. B 108, 17879 (2004).

13 In semiempirical approaches, as in Ref. 3, the conductance follows a simple exponential decrease with the length of the molecule.

14 Core electrons were modeled with Troullier-Martins soft norm-conserving pseudopotentials and the valence electrons were expanded in a SIESTA localized basis set. A double $\zeta+$ polarization basis for the OPVn molecule, while a double $\zeta$ for the gold $s$ channel and single $\zeta$ for the gold $p$ and $d$ channel was used. See Ref. 10 for details. The LDA was used for $\mathrm{XC}$ functional.

15 Y. Xue et al., Phys. Rev. B 59, R7852 (1999).

16 The geometry of the molecules in the junction is assumed to be the same as the isolated molecules, which are obtained at the level of SVWN/6-31+G*; see Ref. 24 .

17 The electrode is modeled as a $3 \times 3$ unit cell, and the $\mathrm{S}$ atoms were placed at $1.87 \AA$ from the fcc position of $\mathrm{Au}(111)$. The cell was big enough to avoid the cross linking of molecules.

18 The results for triyne, pentayne, and heptayne (not shown here) fit the curves for even members of the series.

19 R. Landauer, Z. Phys. B 68, 217 (1987).

20 The effect of the enhanced DOS at $E_{f}$ due to the small interelectrode separation for shorter molecules should also be present here; see Ref. 5 .

21 HOMO and LUMO levels for molecules connected to electrodes refer to the Molecular Projected Self-consistent Hamiltonian (MPSH) levels.

22 J. P. Perdew et al., J. Chem. Phys. 123, 062201 (2005); 
S.J.A. van Gisbergen et al., Phys. Rev. Lett. 83, 694 (1999).

23 The results of such schemes for polarizability and hyperpolarizability of isolated molecules were largely overestimated when compared with methods with more accurate nonlocal
XC potentials. See Ref. 22 .

24 M.J. Frisch et al., Gaussian 03, Gaussian Inc., Wallingford CT 2004. The 6-31+G* basis set was used.

25 M. Weimer et al., Chem. Phys. 309, 77 (2005). 